\title{
Exploring How Tangible Tools Enable Collaboration in a Multi-touch Tabletop Game
}

\author{
Tess Speelpenning ${ }^{1}$, Alissa N. Antle ${ }^{2}$, Tanja Doering ${ }^{3}$, and Elise van den Hoven ${ }^{1}$ \\ ${ }^{1}$ Eindhoven University of Technology, The Netherlands \\ e.v.d.hoven@tue.nl \\ ${ }^{2}$ Simon Fraser University, Surrey, BC, Canada \\ aantledsfu.ca \\ ${ }^{3}$ University of Duisburg-Essen, Germany \\ tanja.doering@uni-due.de
}

\begin{abstract}
Digital tabletop surfaces afford multiple user interaction and collaboration. Hybrid tabletops that include both tangible and multi-touch elements are increasingly being deployed in public settings (e.g. Microsoft Surface, reacTable). Designers need to understand how the different characteristics of tangible and multi-touch interface elements affect collaborative activity on tabletops. In this paper, we report on a mixed methods exploratory study of a collaborative tabletop game about sustainable development. We explore the effects of tangible and multi-touch tools on collaborative activity. Forty-five participants, in trios, played the game using both versions of the tools. Our analysis includes quantitative performance measures, qualitative themes and behavioral measures. Findings suggest that both tangible and multi-touch tools enabled effective tool use and that collaborative activity was more influenced by group dynamics than tool modality. However, we observed that the physicality of the tangible tools facilitated individual ownership and announcement of tool use, which in turn supported group and tool awareness.
\end{abstract}

Keywords: Tangible interaction, collaboration, CSCL, tabletop gaming, multitouch, Futura, interaction design.

\section{Introduction}

Since the concept of graspable user interfaces was first defined by Fitzmaurice and colleagues [1] interactive user interfaces have developed in many areas and are appreciated because of their advantages over traditional mouse and keyboard based interaction. Interactive user interfaces can provide multi-touch and multiple user interaction and opportunities to weave the physical and digital world together in a seamless matter [2]. Although there are many similarities, a multi-touch interface is distinct from a tangible user interface (TUI). Multi-touch interfaces enable "hands-on computing" [3], direct manipulation, joint actions and spatial visibility of movements [3]. In addition to the characteristics of multi-touch interfaces, TUIs may provide affordances that are more natural [4], enable manipulation in 3D space and may 
improve spatial cognition [5]. These differences are also described as embodied facilitation [6].

Technologies such as SMART boards, plasma screens, and projected wall displays all have been studied in order to better understand how to design them to support collaborative interaction [7]. Several different tabletop systems have also been developed to support multi-user interaction that facilitates collaboration (e.g. Microsoft Surface, ReacTable). For designers it is important to understand how the characteristics of these different interface styles affect user experience. Some comparative studies have explored how multi-touch tabletops enable social interaction (e.g. $[8,9,10])$.

Several studies suggest that multi-touch tabletops stimulate more collaboration compared to traditional user interfaces (e.g. [7, 9, 11, 12]). In addition, tangible artifacts that are part of a user interface may invite people to interact more, because tangible objects lower the threshold for participation [13]. Thus, multi-touch interactive tabletops with additional tangible objects may provide new opportunities for socializing, collaborative learning and working. Nevertheless, as Hornecker and Buur [13] stated, the research field lacks an analysis and understanding of the aspects that support the social aspects of tangible interaction. A design framework that provides concepts or guidelines that help designers understand how to support collaboration related to multi-touch-tangible tabletops does not yet exist.

In this paper, we report on findings from an exploratory comparison of multi-touch and tangible controllers for a visualization tool that were developed to augment a collaborative, sustainability learning game, called "Futura: the Sustainable Futures Game" (original game described in [14]). We abbreviate the game title as "Futura" in the remainder of this paper. Our study addressed two research questions: 'How does tool use differ between multi-touch and tangible interaction?' and 'How does the user interface (UI) style of the tools affect collaboration?' Our goal was to understand how the characteristics of the tangible and the digital controllers for the visualization tools affected activity and collaboration in the context of a tabletop game.

The learning goal of Futura is to increase players' awareness of the complexity of sustainability issues. Previous work with Futura indicated that players had trouble linking individual actions to cumulative, global effects [13]. In addition, many of the groups played the game with a parallel play or individual style at the beginning of game sessions, and shifted to a more collaborative play style only after they played the game several times [14]. Therefore, the goal for the visualization tools was to support players to understand cumulative effects and to enable an earlier shift from individual to collaborative play. We implemented visualization tools that addressed these needs and that could be controlled with either tangible or multi-touch objects. This enabled us to compare the tangible and multi-touch controllers of the visualization tools to determine how UI characteristics changed or enabled interaction and collaboration. For brevity we call the tangible and multi-touch controllers that are part of the visualization tools, the "tools". Compared to prior work, this exploratory study focused on a comparison of two versions of visualization tools, which were novel elements in the Futura game. 
Prior research based on the Futura game has included two observational studies focusing on understanding how people learn [14] and a survey-based case study that explores how Futura can be used to engage the public with issues of societal importance [15]. This paper presents a comparative study focusing on how the UI style of these tools affects collaboration. The study was conducted with a redesigned Futura application.

\section{Related Work}

Tabletop-based games have been in the focus of several case studies on interaction with tangible and multi-touch UI elements. Examples of studies of these kinds of hybrid tabletop gaming can be found in [16], [17] and [18]. Few studies explore how the different affordances of tangible or multi-touch interface elements affect interaction and collaboration. There are no existing studies that compare the effects of tangible and multi-touch interaction on collaborative game play activity. In this section, we will summarize some research that compares users' performance and preference using mouse, multi-touch and tangible user interfaces for a variety of application areas.

In a user study in which participants had to implement warehouse layouts with either multi-touch or tangible shelves, Lucchi et al. [19] found that the completion time for the tangible group was faster or more efficient. The authors assumed that this was caused by users' familiarity with physical artifacts in the everyday world. In another user study where users had to do several matching tasks [20], the authors also found that tangible artifacts were easier and more accurate to manipulate. However, they emphasized that effects likely depend on the task, tangible objects and quality of technological implementation.

A user study with children solving jigsaw puzzles using three different UI styles (physical, TUI and GUI) showed no significant differences in children's enjoyment. Observation data indicated that children's engagement was higher for both physical and tangible conditions, compared to the graphical condition [21]. A follow-up study using video analysis of hand-actions indicated that there were significant differences in the frequency, duration and type of actions used to manipulate puzzle pieces between UI styles [22] and that tangible and physical UIs facilitated many fast actions, and a more sophisticated puzzle solving strategy that evolved over time.

Wang [23] performed a comparative study of a tangible and a multi-touch interface in which he investigated the effect of interface style on users' strategies and performance solving a jigsaw puzzle. Both multi-touch and tangible interaction enabled complementary actions (i.e. using physical actions on objects to reduce cognitive load) but interaction was more precise and efficient with the TUI version. The author suggests that these performance benefits may be a result of the tangible puzzle's 3D manipulation space and tactile feedback.

In a study by Rogers and colleagues [24], the authors compared a mouse, multitouch and physical-digital condition for a collaborative design task. The mouse condition led to frustration because it resulted in unequal participation. However, it resulted in the most utterances. The physical-digital condition led to more equitable participation in terms of verbal contribution. The multi-touch condition had the most 
equitable participation in terms of physical actions. Based on their findings, we focus on physical as well as verbal interactions between the users.

\section{Background Theory}

In order to investigate how the characteristics of tangible and multi-touch tools affect collaboration, we first provide a working definition of collaboration. Roschelle and Teasley [25] (page 70) defined collaboration as "a coordinated, synchronous activity that is the result of a continued attempt to construct and maintain a shared conception of a problem". This activity results from individuals that share and negotiate meanings to solve a problem. In this model, collaboration is measured in terms of communication. Brehmer [26] describes the relationship between collaboration and communication as follows: if the necessity for coordination and cooperation increases, the need for communication also increases.

The theory of Computer Supported Collaborative Learning (CSCL) provides an important perspective on collaborative learning which applies to groups playing a learning game, like Futura. A review of the CSCL literature suggests three elements that are required to support collaborative learning: shared objects of negotiation [27], referential anchors [28] and metacognitive processes [29]. In our study, we focus on shared objects of negotiation, because we are interested in characteristics of objects -tools -- that may affect or improve collaboration. We also draw on empirical findings from the tangible interaction literature because designers need to understand how the different characteristics of tangible and multi-touch elements affect collaborative activities on tabletops. We next summarize the following main theoretical concepts from the CSCL and tangible interaction literature that we use to inform our analysis: objects of negotiation, access points, physical constraints and awareness. In our thematic analysis of observational data (below), we also search for emergent concepts.

\subsection{Objects of Negotiation}

Objects of negotiation are objects that externally represent information that is important in some way in the collaborative task [27]. An individual or group can modify this representation during the learning process. If an individual changes a shared representation, he may feel an obligation to announce, discuss or negotiate his action with the rest of the group in advance [27]. In Futura, players must collaborate and therefore communicate about various different external representations in order to win the game and through winning attain the game's learning goal. The tools we developed enabled players to change the main external representation in the game, as described below in section 4.3.

\subsection{Access Points}

Access points are physical or spatial interface characteristics that enable a user to join a group's ongoing interactions with a system. They provide perceptual and manipulative access and enable fluidity of sharing [30]. These characteristics may enable a user to interact, participate and join a group's activity. The amount and type 
of input devices that are used in shareable interfaces are important factors that shape interaction and affect the type of social interaction. Manipulative access and fluidity of sharing are two elements that are amplified by tangibility. The fluidity of sharing describes how easily one user hands over control to someone else [30]. One of the benefits of tangible tools that result from their physical and spatial qualities is that they may better enable access compared to similar multi-touch tools [30].

\subsection{Physical Constraints}

Hornecker [13] describes embodied facilitation as physical and perceptual affordances and spatial properties that shape and determine people's interactions with a physical artifact. Some of the affordances of physical artifacts enable specific actions that are not possible or less obvious when using digital artifacts (e.g. a multi-touch tool). For example, physical or tangible tools can be manipulated in 3D space, whereas the use of multi-touch tools is constrained to 2D space. Because of this, a wider range of actions may be performed with tangible artifacts, and such actions are more visible to other users [23].

\subsection{Awareness}

Awareness is involved in creating a context for a person's activities by understanding the activities of others [31]. Ideally, individual contributions are relevant to group activities in that they support the group to collaborate. Hornecker found that multitouch input resulted in more positive indicators of workspace awareness compared to mouse input in a collaborative task including an increase of helping behaviors and more handovers of objects [32]. She suggested "being able to see another's physical actions can enhance awareness, which in turn can support fluid interaction and coordination" [32] (page 167). Fleck et al. suggest that "maintaining joint attention and awareness" is critical to coordinated, collaborative activity [33]. They suggest that a physical artifact may better enable collaboration because of what we can do with it. For example, a physical artifact draws attention when a user picks it up from a tabletop and moves it in 3D space in front of their body.

\section{The Futura Game and Visualization Tools}

\subsection{Overview}

Futura (Figure 1) is a multiplayer, tabletop game that challenges players to make decisions that help preserve the environment, and at the same time meet the needs of a growing population. There are three roles to be played in the game: providing food, energy or shelter. Each role can access specific resources. Players can place resources on the world map to support the growing population, but they have to make sure that the resources do not damage the environment. The goal of the game is to balance supporting the population and damaging the environment in a simulated world. The game spans many years and the first version of the game lasts about three minutes. For more details about the original game, see $[14,15]$. For a video overview of the Futura project, see http://www.antle.iat.sfu.ca/Futura. 


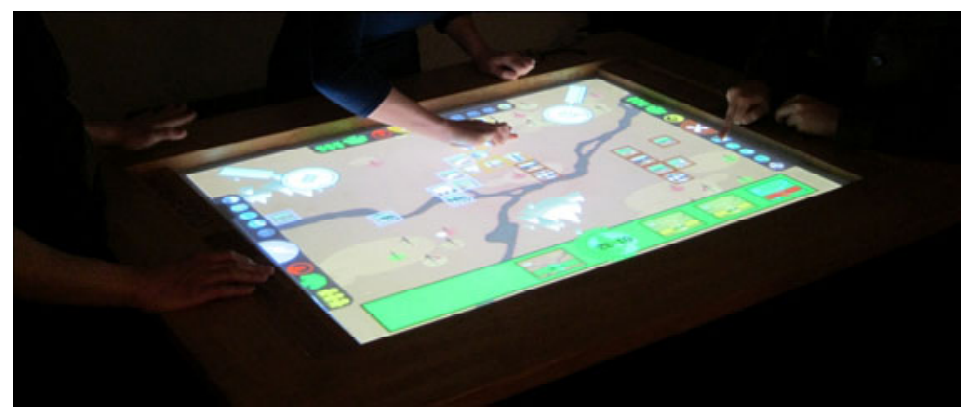

Fig. 1. The Futura game (in the multi-touch condition)

\subsection{Design Goals: The New Version of Futura}

Players can only win the game when they collaborate since all three roles must work together. Coordination and communication are necessary. Based on results of a previous study we wanted to better enable player understanding of the cumulative impact of actions on the world state, and to support collaboration based on this information [14]. We augmented the original version of Futura (described in [14]) with two visualization tools.

\subsection{Visualization Tools}

We developed two visualization layers that provide information about the cumulative impact of player actions on the environment, and on the population. Each of the two layers is activated using magnifying glass tools. There are two tangible magnifying glasses (TUI), and two magnifying glasses implemented as digital objects activated through multi-touch (MT). We refer to these as the TUI and MT conditions. In both conditions, the two tools provide players with more information about the cumulative impact of each resource that has been placed on the world map up to that point in the game (on the environment and population respectively). One of the magnifying tools is labeled with a tree and represents the impact on the environment (Figure 2). The other is labeled with people and represents the ability of the current resources to meet the needs of the population. The impact visualizations are generated in real time based on the current state of the game world. The impact visualizations are in the form of
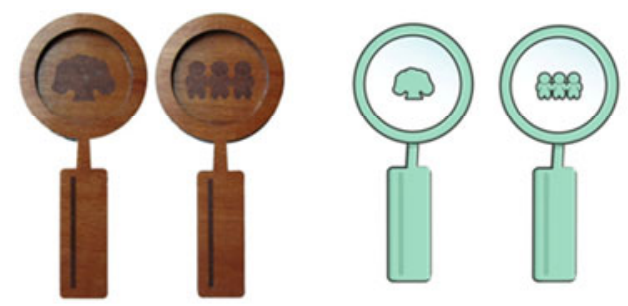

Fig. 2. Tangible (left) and multi-touch (right) magnifying glass tools 
"heat maps" in which colour indicates the strength of effect. We used two slightly different colour mappings for the two maps so they could be differentiated (red/dark pink is hot or negative; green/blue is cool or positive). For example, for the environmental layer, the colour red indicates areas of high damage caused by resources located in those areas. In the population layer, dark pink indicates areas where resources are not efficiently supporting the population.

\subsection{Tool Usage}

When a player uses one of the magnifying glass tools to activate a visualization layer, the game pauses for everyone, and the appropriate impact visualization appears as an overlay on the game world (Figure 3). In the TUI condition, the tangible magnifying glasses are placed on the edge of the table. Any player can place a tool on the table's active surface to activate the corresponding impact visualization. Removing the tool removes the visualization. Only one of the visualizations can be displayed at a time.
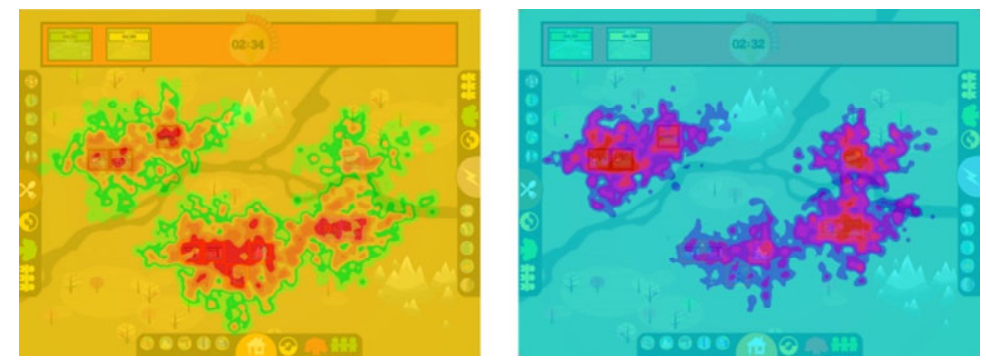

Fig. 3. Impact visualization - environment (left), impact visualization - population (right)

In the MT condition, the two magnifying glasses are static buttons placed in each lower corner of the tabletop. Touching a multi-touch tool button will activate the corresponding visualization. Touching the resume button will remove the visualization. All other game features are identical between the two conditions. The tools may provide opportunities for collaborative communication and coordination. For example, players may use the displayed impact visualization to trigger discussion of the contribution of each resource type to the current game state and then modify their game strategy.

\subsection{System Implementation}

The Futura game runs on a multi-touch version of EventTable, a custom-made digital tabletop system that facilitates both multi-touch and tangible interaction, the latter using the reacTIVision-tracking library [34]. Fiducial markers are used to track the tangible tools. The platform is initially described in [14]. Some modifications were made to improve finger tracking. For example, three cameras were added to the existing camera configuration. This is described in detail in [23]. Fiducial markers are used to track the tangible tools. 


\section{Study Methodology}

In order to understand the differences between tangible and multi-touch tool use, and the effects of tool interface style on collaboration, we designed an exploratory comparative study between a tangible interface (TUI) condition and a multi-touch (MT) condition of the visualization tools for the game Futura. We used a within subjects design, because the group dynamics and personality of users may have a major influence on the results [35]. Another advantage of a within subjects design is that users are able to compare the two UIs. The order of the conditions was counterbalanced (AAABBB or BBBAAA).

\subsection{Participants}

We collected data from 45 participants who played the game in groups of three players resulting 15 different groups. Participants (26 male, 19 female) in our study were predominantly graduate and undergraduate students from different programs at Simon Fraser University (Canada). Ages ranged from 18 to 36 years old $(M=23, S D$ = 3.69). Most participants reported that they were in the third year of their study (14 out of 45). Most participants reported that they played computer games sometimes (16 out of 45) or often (13 out of 45), but had never (22 out of 45) or rarely (17 out of 45) interacted with a digital tabletop.

Participants were recruited through a user study pool at the university. The groups were randomly assigned and thus participants knew each other to different degrees: some were friends (13 out of 45), classmates (16 out of 45), others did not know each other at all (16 out of 45). Participants were rewarded with a $\$ 10$ gift card for participating in our study.

\subsection{Procedure}

To minimize potential learning effects, we explained the mechanics of the game carefully before participants started playing using an in-game tutorial. To stimulate the use of the magnifying glass tools, we demonstrated and encouraged the use of them at the end of the tutorial. Participants played each of the two conditions (TUI and MT) three times. In previous studies, we noticed that many groups played the game about three times before they were able to win (without a tutorial) [14]. They were directed to try to win the game. Each game took about five minutes (longer than the original three minute version of the game because players used the tools to pause the game). The total duration of the study was about 50 minutes for each group. The participants were asked to fill in the game questionnaire after playing in both conditions. The study setup is shown in Figure 4.

\subsection{Data Collection and Analysis}

The mixed methods study design involved collecting data about demographics, game performance, tool use behaviors, player opinions, as well as observations of interaction and play patterns. Quantitative measures included game performance, game scores, and tool use. Tool use data was analyzed for both frequency of 
occurrence and temporal patterns of usage. In the questionnaire, we asked about learning outcomes, collaboration and tools use. For example we asked the participants if they used the magnifying glasses (TUI and MT) to play the game and if they collaborated with others. We also asked participants if they thought the physicality of the tools made a difference. We used both open and closed questions, the latter ranked with a 7-point Likert scale. We analyzed numeric data with Wilcoxon test to compare the difference of two means, since the data involved repeated measures and included non-Normal count data and ordinal Likert ratings.
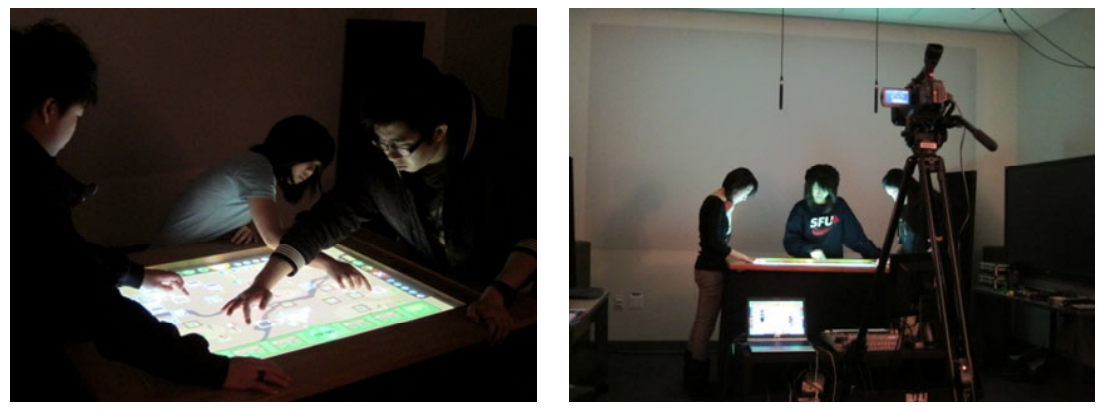

Fig. 4. Playing Futura in groups of three (left). The setup of the user study, showing the Futura game tabletop, three players, microphones, video camera and a additional screen (right).

Since we were interested in how the tangible tools influenced collaboration, we also collected qualitative observational notes and video. Real-time observation of the table surface on an additional screen helped the observers to see what was going on in the game without being intrusive (Figure 4, right). We used a structured note taking approach to record how they interacted and collaborated with each other, when players started interacting, how they used the tools, and how this changed over time. Afterwards three researchers looked for patterns in collaboration. We did this by analyzing our observational notes and video through the lenses of theoretical concepts (objects of negotiation, access points, physical constraints and awareness) as well as searching for other repeated patterns or themes. Based on previous work comparing physical-digital and multi-touch tabletops, in which verbal and physical acts were both deemed important [24], we considered verbal interactions including: utterances, discussion, information exchange, negotiation, and suggestions, and physical interactions including: gesturing and performing actions on game objects.

\section{Results}

Our results address two research questions: 'How does the tool use differ, comparing multi-touch and tangible interaction?' and 'How does the UI style of the tools affect collaboration?' Results provide insights about how players use the tools, how they collaborate with and through the tools, and with each other. We report our general tool use related findings first, then follow with our four theoretical themes: object of negotiation, access points, physical constraints and awareness. We introduce new themes under each of these, as appropriate. 


\subsection{General Tool Use}

We observed that all 15 groups used the environment and population magnifying glasses in both the TUI and MT touch conditions. Groups used the tools during most of their games (72 out of 90 games). In total, we counted 204 magnifier usages in 90 games ( $90=2 \times 3$ games $\times 15$ groups). Of these, 108 usages occurred with the TUI tools and 96 with the MT tools. Neither condition significantly differed regarding frequency of usage (average number of usages per game TUI: 2.4, MT: 2.1). However, the frequency of usage varied from group to group. The maximum number of magnifying glass usages (summed over all six games in both conditions for each group) was 20 and the minimum was three.

Basic usage patterns were similar for the TUI and MT groups. For example, we found that users would normally not use the magnifier glasses early in the first game (in either condition), since they were still focused on understanding their own game roles and possibilities in the game. Sometimes players seemed to forget about the tools, but started to use them later, "Maybe we should try these" (man, age 26, group 2, game 2). Furthermore, if people figured out a strategy to win the game, which normally took at least a few games, they used the tools less, since they did not need the additional information.

We also looked at how long people used the tools and if there were temporal patterns of usage (e.g. using a tool at a specific moment in the game or using both magnifier glasses directly one after each other). We found that early magnifier glass usages (within the first 45 seconds) occurred more in the second condition, regardless of which condition came second (first session $=$ MT 2, TUI 3; second session $=$ MT 8 , TUI 10). The pattern of using both magnifier glasses shortly after each other occurred more often when players played their first three games with the TUI tools. We suggest that this could indicate that the TUI tools were more suitable for shared explorations. This suggestion needs further research.

\subsection{Object of Negotiation}

The tools affected the game play of everyone in the game when they were used. Our analysis of qualitative observational data indicated that people used the tools in different ways independent of condition. For example, some players only quickly glanced at the impact visualizations without having a conversation and others explored the impact visualizations in depth but did not talk. Others used it to discuss either game functionality or game strategy, sometimes including gestures like pointing to specific points on the visualization and thus, focusing all players' attention to this spot. We observed these different behaviors equally between players in both conditions, and we did not find any significant differences in how they used the tools to negotiate with each other or play the game. The characteristics and quality of collaboration we observed depended primarily on the group dynamics and personality of individuals. As can be seen in Figure 5, some groups communicated and interacted frequently while other groups played the game largely individually. The differences in group dynamics can be explained by the different relationships in groups: some players knew each other well, others were strangers; some groups consisted of quiet and shy people and other groups had one or two main "talkers". The finding that group dynamics is an influencing factor is in line with findings from other studies [12, $24,35]$. 
Analysis of the questionnaire data indicated that although users tended to like the second tool they used more, many preferred the TUI tools regardless of if they used them in the first or section sessions (26 vs. 17). This contradiction between use and preference may be explained by the learning effect of the game: when players understood the game better, they were more likely to win and thus liked everything about the game more (including the tools). We did not see differences in collaboration responses in the questionnaire data. For example, the Likert rating for the statement "The magnifying glasses helped me to collaborate with other players" was similar in both conditions $(\mathrm{Z}=-.938$, n.s. $=.348)$. One person said he would like to have tools for everyone individually.

In both conditions and in both session orders, players used the environment tool more than the population tool (60\% vs. $40 \%)$. We suggest that players may have understood the environment impact visualization better than the population one. In the environment impact visualization, the colour red represented damage, which was easily understandable for all users, "See, here where the marine resource is, it's less polluted" (woman, 22, group 12). However, the population impact visualization showed how well the population was supported. Dark pink meant that the population was not well supported. This was not always clear, "we need more population" (man, 22 , group 15). Other players related the population impact visualization to density.
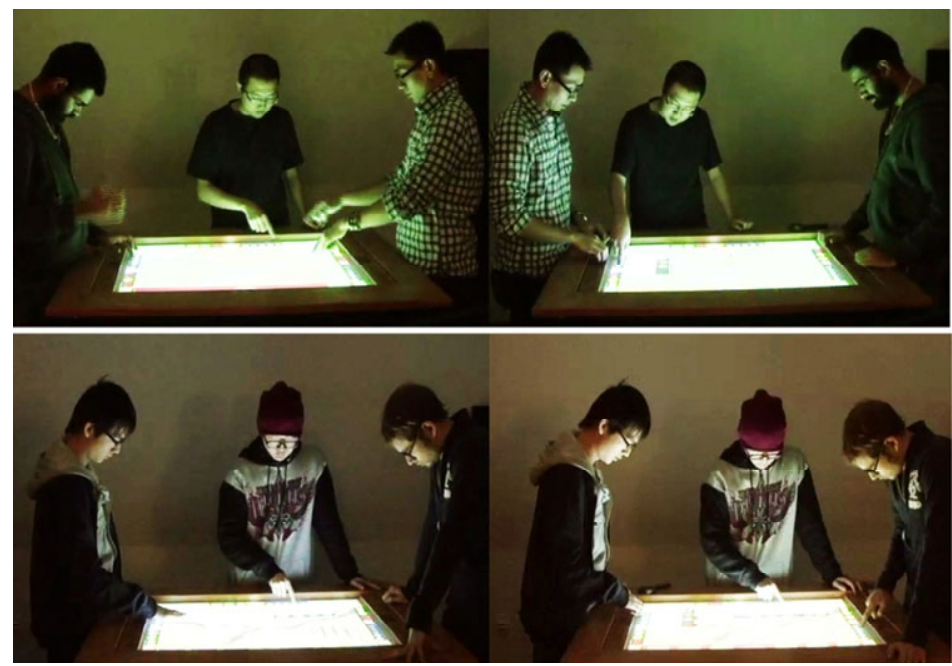

Fig. 5. Group dynamics; collaborating group (on top) and individual play (bottom), in both conditions (MT left, TUI right)

\subsection{Access Points}

We would expect a difference between the conditions related to access points, because the physicality of tangible tools enables mobility and handing over or passing around the tools, which could have influenced collaboration. However, our results showed no differences in how players accessed the game in either condition. This may be because all three players started the game together. The effect of access points may be more important when an additional player is joining an existing game. 


\subsection{Physical Constraints}

In the TUI condition, we observed that initiating and resuming of the impact visualizations were done significantly more often by the same person than in the MT condition; $77,4 \%$ vs. $56 \%$ (Wilcoxon test; $\mathrm{Z}=-5.83, p<.001$ ). In the MT condition, a different person more often resumed the game by touching the resume button in the middle of the screen. However, this does not suggest more collaboration because it often happened without the resuming player checking with the other players. In the MT group, players often reached over and canceled the impact visualization that someone else activated, without clear announcement or communication (Figure 6). This may have occurred less in the TUI group because of the physical and spatial constraints on the actions that can be taken with a physical object [13].

This finding can be explained by looking to meaning rather than behaviors alone. Each type of action that is enabled by an artifact's affordances can change the meaning of the artifact. Physicality opens up meaning making. We suggest that the finding that many more players resumed the game themselves in the TUI condition may be explained the concept of ownership. The physicality of the tool enables the action of picking it up and holding it, which may in turn cause a feeling of ownership. Psychological ownership is defined as the state in which individuals feel an object is theirs [37]. Thaler [36] uses the term "endowment effect" to describe the idea that goods that belong to one's endowment are valued higher than identical goods that do not. Endowment contributes to a feeling of ownership. When a player picks up and holds one of the TUI magnifying tools, they, and other players, may all sense that the object temporarily belongs to that player. Other players may then hesitate to take action on the object which was placed by, and temporarily owned by, another player. Further investigation is needed to find out whether it is this sense of ownership that inhibits the initiating and resuming of other players, when using physical tools.
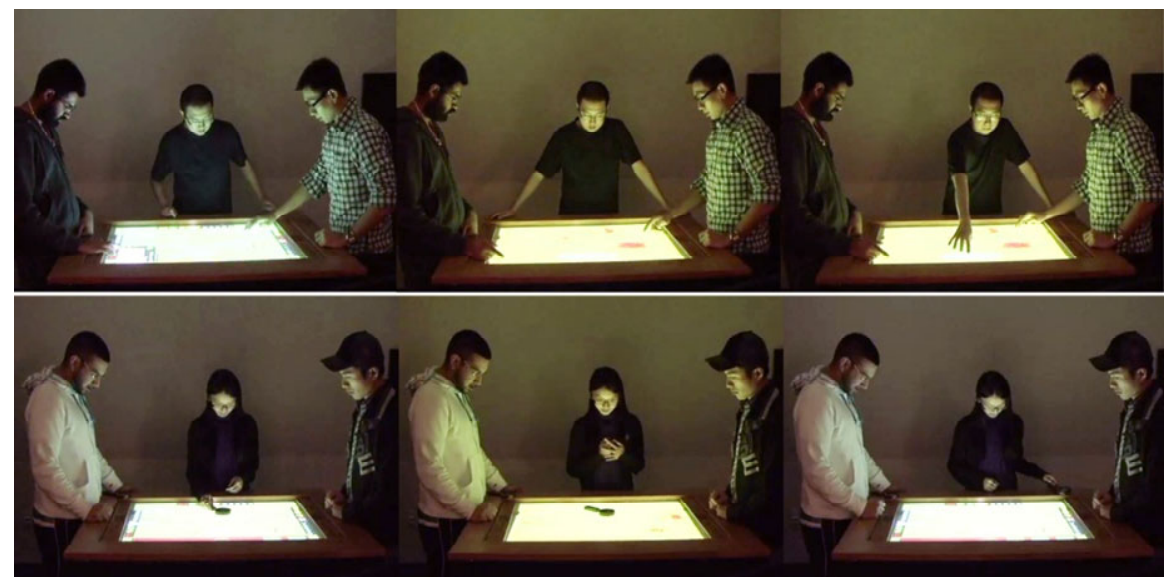

Fig. 6. Initiating and resuming tools is more often done by different players in the MT condition (top); and more often done by the same player in the TUI condition (bottom). For both conditions, from left to right: 1) Player activates impact visualization; 2) Impact visualization is activated; 3) Player resumes the game. 


\subsection{Awareness}

Our analysis of observational data indicated that players more often announced that they were going to use one of the tools in the TUI condition compared to the MT condition. Announcements can be verbal, gestural or take the form of actions on objects. For example, announcements include visibly holding up a physical tool or waiting for others to finish their tasks before using a tool.

Using the tools affected the game play of everyone and so an individual may have felt an obligation to obtain agreement from the other players before using a tool. In our analysis, we found more instances of gestures, eye contact, and verbal announcements in the TUI condition. This may be because tangible interaction in 3D space makes individual actions more legible, which leads to better peripheral awareness. People need to make body movements in order to use both the multi-touch and the TUI magnifying tools. However, the 3D interaction space and the physicality of the TUI tools make the use more visible when they are lifted off the table in preparation for placement or to resume play [23]. This visibility may have made people more aware of each other and the tools (Figure 7). When asked why they liked the TUI tools more, some users (6 out of 26) said that they liked being aware of using a physical artifact.
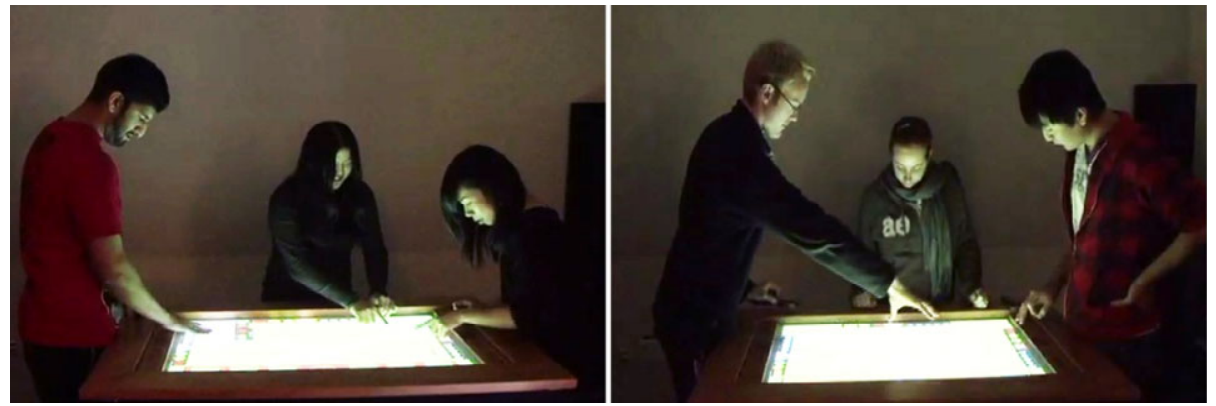

Fig. 7. No tool usage announcing in MT condition (left), clear gesture of tool usage announcement in TUI condition (right)

Awareness contributes to group coordination and collaborative context [31]. Coordination of actions is an important part of collaborating [32]. Announcing the use of the tools may contribute to the coordination of collaboration [33]. In a collaborative learning game, individual contributions are relevant to group activities. Awareness is critical when designing a collaborative system [31] and announcing the use of the TUI tools can be seen as an increasing awareness about the game. Gestures and utterances make it clear that people are going to use the tools. The findings that players in the TUI condition more often activated the visualization, that the same person more often resumed the game, and players more frequently announced tool use support the suggestion that the TUI design better enables tool awareness. We suggest that these findings support the effectiveness of the TUI tools for enabling awareness. Enhancing awareness supports fluid interaction and coordination during collaboration. 


\subsection{Limitations}

The tools we added to the game are not mandatory to win the game. Although all groups used the tools at least once, it may be possible that players did not think they were useful and decided not to use them frequently. In some cases, the players linked information to the impact visualization that was not related at all. For example, some players interpreted the game world and impact visualization as location specific "maybe we can put highly polluted things far away" (woman, 27, group 7). While the impact map shows the impact of each resource at the location where the resource has been placed, the spatial locations of resources on the map do not mediate their impact in any way. For example, placing a highly polluting resource anywhere on the map would affect the game state in the same way. This possibly confused players. Furthermore, since we did not test the effectiveness of the impact visualizations, it is possible they were confusing instead of helpful. However, any negative effects would have occurred in both conditions.

The tangible object recognition system (using fiducial markers) did not work as precisely as the touch recognition of the system. This means that activation and deactivation of the visualizations using the multi-touch (buttons) was easier and faster than using the TUI objects. This may explain why $17 / 45$ participants liked the multitouch tools better. Of these participants who liked the multi-touch tools more, all of them gave these reasons: the MT tools worked better, faster or were easier to use. There was also some latency between activating a tool and the appearance of the impact visualization (about 0.5s). Although the latency was present in both conditions, it was slightly longer in the TUI condition. Neither of these limitations appears to directly impact our results about ownership or group and tool awareness.

Generalization of our results should take into account that we performed this study in a lab setting. For example, this limits our ability to make inferences about tool use, collaboration and public engagement in a public setting. In addition, the statistical conclusion validity is limited because of the small sample size of 15 groups.

\section{Conclusions}

In this paper, we present the results of an exploratory study that compared the differences between tangible and multi-touch tool use, and the impact of tool use on collaboration in a digital tabletop game. Analysis of tools use and its impact on collaboration was viewed through a concept from the theory of CSCL: objects of negotiation, and concepts from previous empirical research in tangible interaction: access points, physicality and awareness. Research that includes analysis using theories about collaborative learning and previous findings from tangible interaction studies for tabletop game applications is one of the unique contributions of our work.

This paper presents new and valuable insights about tool use, objects of negotiation, access points, physical constraints and awareness in collaborative tabletop settings. We provide insights about how people participate and interact with both tangible and multi-touch tools in the context of a tabletop game. Our gamespecific findings include that the tools were used in different ways regardless of UI style. Some players used it individually, through quiet pondering, and others used the 
tools intensively and collaboratively to helped them to discuss the game strategy. We also found that players in the MT group often "took over" tool usage that a different player had initiated without consultation. We suggest that the $3 \mathrm{D}$ interaction space and physicality the TUI tools may have contributed to the feeling of psychological ownership which prohibited this from happening as much in the TUI condition. This is supported by the finding that tangible tool use was correlated with more player announcements (both verbal and behavioral). We suggest that better awareness of group activity, and therefore better collaboration, can occur when the same player activates the visualization and resumes the game when this is accompanied by some form of announcement. In terms of player preferences, we found that, despite implementation limitations, players still preferred the tangible tools.

Overall, our results provide limited support for the benefits of tangibility. Physical affordances can change the meaning of the artifact as well as actions taken with it. We introduced the concept of ownership which we used to explain player behaviors in the TUI condition. The physicality of the tools and the 3D interaction space may enhance ownership, enable announcements of use, and facilitate awareness. Together these processes and forms of interaction support coordinated collaborative activity. We presented the details of our analysis to support designers to make decisions about tangible and multi-touch elements of digital tabletops deployed to facilitate collaborative activity.

Acknowledgements. The authors would like to thank Sijie Wang and Nahid Karimaghalou for their technical support, Anna Macaranas, Allen Bevans, Katie Seaborn and Theresa Jean Tanenbaum for their ideas, thoughts and practical support. Thanks to Wijnand IJsselsteijn for his coaching. In addition, we would like to thank all the participants of our user study for their participation. This research was funded by the NSERC RTI and the GRAND NCE grants from Canada.

\section{References}

1. Fitzmaurice, G., Ishii, H., Buxton, W.: Bricks: Laying the Foundations for Graspable User Interfaces. In: CHI 1995, Denver, CO, USA, pp. 442-449. ACM Press, New York (1995)

2. Ullmer, B., Ishii, H.: Emerging Frameworks for Tangible User Interfaces. In: HumanComputer Interaction in the New Millenium, pp. 579-601. Addison-Wesley, Reading (2001)

3. Kirk, D., Sellen, A., Taylor, S., Villar, N., Izadi, S.: Putting the Physical into the Digital: Issues in Designing Hybrid Interactive Surfaces. In: BSC HCI 2009, Cambridge, UK, pp. 35-44 (2009)

4. Jordà, S., Geiger, G., Alonso, M., Kaltenbrunner, M.: The reacTable: Exploring the Synergy between Live Music Performance and Tabletop Tangible Interfaces. In: TEI 2007, Baton Rouge, LA, USA, pp. 139-146. ACM Press, New York (2007)

5. Maher, M., Kim, M.: Do Tangible User Interfaces Impact Spatial Cognition in Collaborative Design? In: Luo, Y. (ed.) CDVE 2005. LNCS, vol. 3675, pp. 30-41. Springer, Heidelberg (2005)

6. Hornecker, E.: A Design Theme for Tangible Interaction: Embodied Facilitation. In: ECSCW 2005, pp. 23-43. Springer, New York (2005) 
7. Rogers, Y., Rodden, T.: Configuring Spaces and Surfaces to Support Collaborative Interactions. In: Public and Situated Displays, pp. 45-79. Kluwer Publishers, Dordrecht (2003)

8. Waldner, M., Hauber, J., Zauner, J., Haller, M., Billinghurst, M.: Tangible Tiles: Design and Evaluation of a Tangible User Interface in a Collaborative Tabletop Setup. In: CHI 2006, Sydney, Australia, pp. 151-158. ACM Press, New York (2006)

9. Scott, S., Carpendale, S.: Guest Editors' Introduction: Interacting with Digital Tabletops. In: IEEE Computer Graphics and Applications, vol. 7(4), pp. 24-27. IEEE Press, New York (2006)

10. Rekimoto, J., Ullmer, B., Oba, H.: DataTiles: A Modular Platform for Mixed Physical and Graphical Interactions. In: CHI 2001, Seattle, WA, USA, pp. 269-276. ACM Press, New York (2001)

11. Price, S., Falcao, T., Sheridan, J., Roussos, G.: The Effect of Representation Location on Interaction in a Tangible Learning Environment. In: TEI 2009, pp. 85-92. ACM Press, New York (2009)

12. Piper, A., O'Brien, E., Morris, M., Winograd, T.: SIDES: A Cooperative Tabletop Computer Game for Social Skills Development. In: CSCW 2006, Banff, Canada, pp. 110. ACM Press, New York (2006)

13. Hornecker, E., Buur, J.: Getting a Grip on Tangible Interaction: A Framework on Physical Space and Social Interactions. In: CHI 2006, Montréal, Canada, pp. 437-446. ACM Press, New York (2006)

14. Antle, A.N., Bevans, A., Tanenbaum, T.J., Seaborn, K., Wang, S.: Futura: Design for Collaborative Learning and Game Play on a Multi-touch Digital Tabletop. In: TEI 2011, Fungchal, Portugal, pp. 93-100. ACM Press, New York (2011)

15. Antle, A.N., Tanenbaum, T.J., Tanenbaum, K., Bevans, A., Wang, S.: Balancing Act: Enabling Public Engagement with Sustainability Issues through a Multi-Touch Tabletop Collaborative Game. In: Campos, P., et al. (eds.) INTERACT 2011, Part II. LNCS, vol. 6947, pp. 194-211. Springer, Heidelberg (2011)

16. Magerkurth, C., Memisoglu, M., Engelke, T., Streiz, N.: Towards the Next Generation of Tabletop Gaming Experiences. In: Proc. of Graphics Interface, Waterloo, Canada, pp. 7380 (2004)

17. Dang, C.T., Straub, M., André, E.: Hand Distinction for Multi-Touch Tabletop Interaction. In: ITS 2009, Banff, Canada, pp. 101-108 (2009)

18. Leitner, J., Haller, M., Yun, K., Woo, W., Sugimoto, M., Inami, M., Cheok, A., BeenLirn, H.: Physical Interfaces for Tabletop Games. Computers in Entertainment, vol. 7(4), pp. 1-21. ACM Press, New York (2009)

19. Lucchi, A., Jermann, P., Zufferey, G., Dillenbourg, P.: An Emperical Evaluation of Touch and Tangible Interfaces for Tabletop Displays. In: TEI 2010, Cambridge, MA, USA, pp. 177-184. ACM Press, New York (2010)

20. Tuddenham, P., Kirk, D., Izadi, S.: Graspables Revisited: Multi-Touch vs. Tangible Input for Tabletop Displays in Acquisition and Manipulation Tasks. In: CHI 2010, Atlanta, Georgia, USA, pp. 2223-2232. ACM Press, New York (2010)

21. Xie, L., Antle, A.N., Motamedi, N.: Are Tangibles More Fun? Comparing Children's Enjoyment and Engagement Using Physical, Graphical and Tangible User Interfaces. In: TEI 2008, Bonn, Germany, pp. 191-198. ACM Press, New York (2008)

22. Antle, A.N.: Exploring How Children Use their Hands to Think: An Embodied Interactional Analysis. Behaviour and Information Technology, http://www.antle.iat.sfu.ca/Physicality/ThinkingWithHands (accepted) 
23. Wang, S.: Comparing Tangible and Multi-touch Interfaces for a Spatial Problem Solving Task. Masters Thesis. Simon Fraser University, Surrey, BC, Canada (2010), https://theses.lib.sfu.ca/thesis/etd6352

24. Rogers, Y., Lim, Y., Hazlewood, W., Marshall, P.: Equal Opportunities: Do Shareable Interfaces Promote More Group Participation Than Single User Displays? Human Computer Interaction 24(1/2), 79-116 (2009)

25. Roschelle, J., Teasley, S.: The Construction of Shared Knowledge in Collaborative Problem Solving. In: CSCL 1995, Berlin, Germany, pp. 69-197 (1995)

26. Brehmer, B.: Distributed Decision Making: Some Notes on the Literature. In: Rasmussen, J., Brehmer, B., Leplat, J. (eds.) Distributed Decision Making: Cognitive Models for Cooperative Work. John Wiley \& Sons, New York (1991)

27. Suthers, D.: Representational Guidance for Collaborative Learning. Artificial Intelligence in Education, 3-10 (2003)

28. Clark, H., Brennan, S.: Grounding in communication. In: Resnick, L.B., Levine, J.M., Teasley, S.D. (eds.) Perspectives on Socially Shared Cognition, pp. 127-149. American Psychological Association, Washington, DC, USA (1991)

29. Duffy, T., Dueber, B., Hawley, C.: Critical Thinking in a Distributed Environment: A Pedagogical Base for the Design of Conferencing Systems. In: Electronic Collaborators: Learner-Centered Technologies for Literacy, Apprenticeship, and Discourse, pp. 51-78. Lawrence Erlbaum Associates, Mahwah (1998)

30. Hornecker, E., Marshall, P., Rogers, Y.: Entry and Access - How Shareability Comes About. In: DPPI 2007, Helsinki, Iceland, pp. 328-342 (2007)

31. Dourish, P., Bellotti, V.: Awareness and Coordination in Shared Workspaces. In: CSCW 1992, Toronto, Canada, pp. 107-114. ACM Press, New York (1992)

32. Hornecker, E., Marshall, P., Sheep Dalton, N., Rogers, Y.: Collaboration and Interference: Awareness with Mice or Touch Input. In: CSCW 2008, San Diego, CA, USA, pp. 167176. ACM Press, New York (2008)

33. Fleck, R., Rogers, Y., Yuill, N., Marshall, P., Carr, A., Rick, J.: Actions Speak Loudly with Words: Unpacking Collaboration Around the Table. In: ITS 2009, Banff, Canada, pp. 189-196 (2009)

34. Kaltenbrunner, M.: ReacTIVision and TUIO: A Tangible Tabletop Toolkit. In: ITS 2009, Banff, Canada, pp. 9-16 (2009)

35. Meerbeek, B., Bingley, P., Rijnen, W., Hoven van den, E.: Pipet: A Design Concept Supporting Photo Sharing. In: NordiCHI 2010, Reykjavik, Iceland (2010)

36. Thaler, R.: Toward a Positive Theory of Consumer Choice. Journal of Economic Behavior \& Organization 1(1), 39-60 (1980)

37. Beggan, J.: On the Social Nature of Nonsocial Perception: The Mere Ownership Effect. Journal of Personality and Social Psychology 62(2), 229-237 (1992) 